\title{
Una mirada, distintas propuestas: género y políticas públicas $^{1}$
}

\author{
Sara MARTÍN BARDERA \\ Universidad de Salamanca \\ bardera@usal.es
}

Recibido: Junio 2015

Aceptado: Febrero 2016

\begin{abstract}
RESUMEN
En este artículo se hace un enfoque analítico de lo que ha supuesto incorporar el concepto de género, desde las teorías feministas, a las políticas públicas. Para ello, se establecen los puntos esenciales de un debate que sigue abierto como problema político y social, como pone de manifiesto la crisis económica iniciada en 2007. Antes de ese momento, en España, las demandas feministas se habían incorporado a la agenda pública como un proyecto integral, caracterizado por la adopción de la perspectiva de género y el "gender mainstreaming". Sin embargo, la crisis muestra la debilidad de las políticas de género en contraste con la fortaleza alcanzada por el pensamiento feminista. En este sentido, se hace una revisión filosófica de sus tesis fundamentales y las consecuencias que deben tener en sociedades que se pretenden más democráticas y, en definitiva, más justas.
\end{abstract}

Palabras claves: Concepto de género, Teorías feministas, Políticas de igualdad.

\section{A view, different proposals: Concept of gender and public policy}

\begin{abstract}
This article analyzes the meaning of "gender" as a category of analysis in public policy. The concept has been transferred from the feminist theories and this has meant that the United Nations and European Union have incorporated the inequality as a structural inequality and an issue justice. So, the feminist demands enter the political agenda as an integral project which is characterized by the adoption of the gender perspective and its application from a transversal methodology ("gender mainstreaming"). In this sense, the "gender ideology" is a new paradigm against the "patriarchal ideology". Now, political actions should be articulated in a double movement of correction and promotion to achieve real equality in societies more democratic and ultimately more just.
\end{abstract}

Keywords: Gender. Feminist Theories. Equality Policies.

${ }^{1}$ Este artículo se publica en el marco del proyecto de investigación: "Bioética y ciudadanía (V): Deliberación estructurada: Análisis bioético en marcos institucionales" (FFI2015-63500-R) financiado por el Ministerio de Economía y Competitividad, dirigido por la Prof. Dra. Ma Teresa López de la Vieja a quién agradezco además las sugerencias realizadas para la elaboración del mismo. 


\section{INTRODUCCIÓN}

"My definition of gender has two parts and several subsets. They are interrelated but must be analytically distinct. The core of the definition rests on an integral connection between two propositions: gender is a constitutive element of social relationships based on perceived differences between the sexes, and gender is a primary way of signifying relationships of power" (Scott, 1986: 1067)

"Género" es un concepto clave en las teorías feministas del último cuarto del siglo $\mathrm{XX}$, sobre todo porque impulsa el desarrollo de unas políticas de igualdad de alcance no equiparable con ninguna acción emprendida anteriormente por los poderes públicos. Este avance no sólo ambiciona traspasar ámbitos temáticos y niveles de gobierno, sino también fronteras. Tal es así que la igualdad de género se postula con carácter internacional, como muestran las conferencias mundiales sobre la mujer y la labor de Naciones Unidas desde ONU Mujeres, al tiempo que demanda la implicación de los estados. También la Unión Europea incluye la igualdad entre mujeres y hombres entre los valores que marcan la política exterior con otros países y organizaciones ${ }^{2}$. En España las políticas de igualdad se inician a partir de la Transición democrática y cobran impulso en la primera década del 2000, en el régimen de género que se establece durante el gobierno de José Luis Rodríguez Zapatero (Lombardo y León, 2014: 16-23).

En este artículo se hace una revisión filosófica de las consecuencias que tiene transponer el concepto de género a la agenda pública. Este traslado condiciona la respuesta a algunas preguntas elementales como ¿qué se entiende por igualdad entre mujeres y hombres? o ¿cómo abolir esta desigualdad? A fin de contextualizar qué significa la igualdad como igualdad de género, hay que tener en cuenta el marco teórico feminista (tabla 1). Éste se establece trazando una línea de continuidad entre el feminismo ilustrado, el feminismo de la igualdad y las reivindicaciones feministas sobre la necesidad de nuevas políticas públicas. El tema se sitúa sobre tres puntales: (1) las ideas de igualdad y diferencia como vertebradoras de un debate que inicia el feminismo ilustrado contra el naturalismo y que es previo a la incorporación del concepto de género; (2) la aportación del concepto al feminismo; (3) las repercusiones del concepto en políticas públicas (tabla 2).

En el campo de las políticas públicas hay que reconocer el amplio desarrollo legislativo e institucional en todos los niveles de gobierno, así como la preeminencia de un discurso público favorable a la igualdad de género. Para ello, a partir del examen del acervo documental y normativo de Naciones Unidas y la Unión Europea (Martín, 2015: 107-174), se pueden establecer dos premisas: (1) que los poderes del estado asumen, que las relaciones sociales se basan en el dimorfismo sexual y son jerárquicas: el hombre domina a la mujer; y (2) que las diferencias entre mujeres y hombres tienen carácter estructural y suponen un problema de justicia. Esta manera de interpretar la desigualdad que conceptualizó Scott (1986), tiene efectos en la forma de abordarla para

\footnotetext{
2 Tratado de la Unión Europea (2012: art. 21.1)
} 
conseguir la igualdad real. ¿Cuáles son? En síntesis, adoptar la perspectiva de género y definir una estrategia adecuada al carácter estructural de la desigualdad: el gender mainstreaming. La consecuencia más relevante es que, por primera vez, las demandas feministas entran en la agenda pública como un proyecto integral.

Sin embargo, la crisis económica que se inicia en 2007, junto con la respuesta dada por la Unión Europea y por los gobiernos de España a partir de $2008^{3}$, muestran una debilidad en la práctica política que contrasta con la fortaleza teórica alcanzada por el pensamiento feminista. Los retrocesos son un indicador de que la igualdad de género no ha permeado las estructuras sociales, de ahí el interés en establecer los puntos esenciales de un debate que sigue abierto como problema político y social.

Tabla 1: Género y teorías feministas. Temas, autoras y propuestas

\begin{tabular}{|c|c|}
\hline \multicolumn{2}{|c|}{ Naturalismo y biología como excusa para la exclusión } \\
\hline $\begin{array}{l}>\text { Mary Wollstonecraff } \\
>\quad \text { Simone De Beauvoir }\end{array}$ & $\begin{array}{l}\Rightarrow \text { Mujer ciudadana } \\
\Rightarrow \text { Mujer: “ser para sí” (ontología) }\end{array}$ \\
\hline \multicolumn{2}{|c|}{ La condición femenina y el problema sin nombre } \\
\hline$>$ Betty Friedan & $\Rightarrow$ Desmontar la "mística de la feminidad" \\
\hline \multicolumn{2}{|c|}{ La otra voz o cómo reconocer la diferencia sin esencializarla } \\
\hline$>$ Carol Gilligan & $\Rightarrow$ Ética del cuidado \\
\hline \multicolumn{2}{|c|}{ El sistema sexo-género (normatividad) } \\
\hline$>$ Gayle Rubin & $\Rightarrow$ Sociedad sin género, no sin sexo \\
\hline \multicolumn{2}{|c|}{ Límites del género (performatividad) } \\
\hline$>\quad$ Judith Butler & $\begin{array}{l}\Rightarrow \text { Genealogía de la ontología del género: sexo como } \\
\text { construcción del género. }\end{array}$ \\
\hline \multicolumn{2}{|c|}{ Género como categoría de análisis y relaciones significantes de poder } \\
\hline$>$ Joan Scott & $\begin{array}{l}\Rightarrow \text { Epistemología feminista y proyección en políticas } \\
\text { públicas }\end{array}$ \\
\hline \multicolumn{2}{|c|}{ Justicia y democracia ampliadas } \\
\hline$>$ Linda Alcoff & $\Rightarrow$ No hay mujer esencial, sino contexto y posición \\
\hline$>$ Seyla Benhabib & $\begin{array}{l}\Rightarrow \begin{array}{l}\text { El otro concreto y el déficit del modelo de justicia } \\
\text { universal }\end{array}\end{array}$ \\
\hline$>$ Nancy Fraser & $\Rightarrow$ Diversidad de públicos: incluir las desigualdades \\
\hline$>$ Chantal Mouffe & $\begin{array}{l}\Rightarrow \text { Fin del agente social homogéneo en una } \\
\text { democracia radical (pluralidad de posiciones y } \\
\text { discursos) }\end{array}$ \\
\hline$>$ Susan Möller Okin & $\Rightarrow$ Justicia y desarrollo moral en la familia \\
\hline
\end{tabular}

\footnotetext{
${ }^{3}$ Véase el análisis de Paleo y Alonso (2014) y Alonso (2015).
} 


$>$ Iris M. Young $\quad \Rightarrow \begin{aligned} & \text { Justicia social, más allá de la justicia distributiva. } \\ & \text { Ámbito público heterogéneo }\end{aligned}$

Fuente: elaboración propia a partir de las obras de las autoras de referencia.

En este artículo se trata de sistematizar qué aporta el concepto de género cuando se traslada como perspectiva a las políticas públicas de igualdad entre mujeres y hombres ${ }^{4}$. La importancia del concepto radica en que ayuda a mostrar que la esfera pública no es imparcial, y ello porque la diferencia afecta a todas las estructuras política, económica, cultural, educativa- en las que los individuos se socializan. Hay que tener en cuenta que las relaciones entre los sexos no empiezan y acaban en cada trato establecido entre los sujetos (Pateman, 1995: 214-259), sino que, como relaciones de dominio y sujeción, se establecen históricamente y se fomentan en el contexto social y cultural. Este contexto forma el sistema sexo-género, donde son ubicados los individuos desde el nacimiento y, en este lugar, los significados y valores son distintos para hombres y mujeres (Rubin, 1986). Uno de los problemas es que ambos sexos asumen, bajo la idea de la complementariedad, esta realidad disociada y ambos participan en su mantenimiento (Bordieu, 2007; Millet, 1995).

Tabla 2: Aportación del debate feminista a las políticas de igualdad

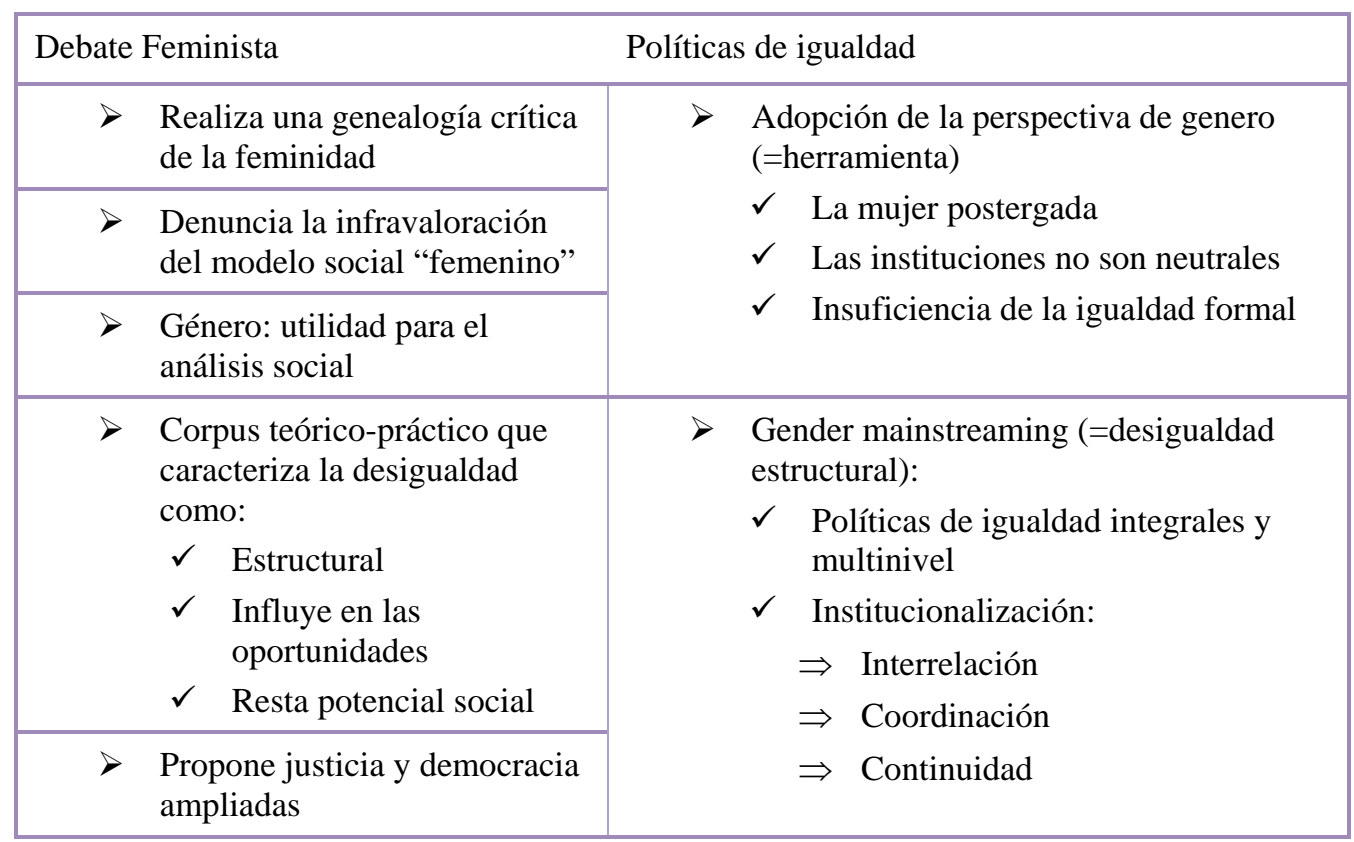

Fuente: elaboración propia a partir de Martín (2015).

${ }^{4}$ De aquí en adelante, por “políticas públicas de igualdad” se entenderá por defecto "políticas públicas de igualdad entre mujeres y hombres”. 
El ejercicio crítico consiste en desvelar las diferentes consecuencias que tiene en las oportunidades y, en definitiva, en la vida de los individuos. Los estereotipos, la segregación en el ámbito laboral, la elección de los estudios y las profesiones, el reparto de las tareas relacionadas con el cuidado, o la distribución del uso del tiempo, son algunas de sus manifestaciones. Si se habla de violencia de género, se está ante la máxima expresión de esas relaciones de dominio y subordinación. Por eso, abordar la desigualdad de género desde la esfera política exige no sólo intervenir en los efectos, sino atender a las causas últimas que la producen e implicar a todos los agentes, también a los hombres.

A continuación se acota el significado de la perspectiva de género en políticas públicas, lo que lleva a desglosar varios aspectos: (1) qué se entiende por políticas de igualdad de género, (2) qué significa la perspectiva de género, (3) cuáles son sus tesis, (4) algunas consecuencias.

\section{1. ¿QUÉ SE ENTIENDE POR POLÍTICAS DE IGUALDAD DE GÉNERO?}

Las políticas públicas son "aquellos productos institucionalizados del sistema político” (Alcántara, 1995: 61). De acuerdo con esta definición, el sistema político está conformado por el régimen político, los actores institucionalizados, la cultura política y el entramado internacional. Es interesante desglosar cada elemento para entender el alcance de la definición, así como tener en cuenta que hay interacciones entre ellos. Esto es necesario para explicar lo que supone la asimilación de la perspectiva de género en políticas públicas.

El régimen político es la estructura formal $\mathrm{y}$, como tal, incluye todos los elementos institucionales, esto es, los poderes públicos y las reglas que establecen las relaciones entre dichos poderes y con la sociedad. Si en algo coinciden las teorías feministas es en considerar que la democracia es el régimen político necesario para el desarrollo de las políticas de igualdad. Aquí estarían las leyes y la administración pública como burocracia y como proceso de generación de productos ${ }^{5}$.

Los actores institucionalizados son los responsables de canalizar las demandas de la sociedad para influir en el gobierno: partidos políticos, grupos de presión y movimientos sociales. Los movimientos y asociaciones de mujeres, a través de su participación en foros como las conferencias mundiales de la mujer, han contribuido a

${ }^{5}$ Acerca de la relación entre administración pública y política, véase Alcántara (1995: 109115): la administración pública sería un instrumento de la política que, al tiempo que la hace posible como acción, la limita. Es algo que está en su origen no sólo para evitar el acaparamiento de cargos públicos por parte del partido vencedor en unas elecciones, sino para garantizar que el sistema siga funcionando en los cambios de gobierno. Hay otro límite que surge de la contraposición entre los valores que rigen en la administración pública y en la política: predicción y eficiencia para la primera, frente a adaptabilidad y respuesta inmediata para la segunda. 
trasladar las demandas del feminismo militante a la agenda gubernamental ${ }^{6}$. También algunos partidos políticos han sido proactivos al incluir en sus programas medidas para favorecer la presencia de las mujeres en sus estructuras ${ }^{7}$, o acciones legislativas relacionadas con la igualdad ${ }^{8}$.

La cultura política tiene que ver con los valores de los individuos y los grupos en cada momento histórico. En este sentido, se puede afirmar que en las sociedades occidentales la igualdad entre hombres y mujeres es actualmente un valor incorporado como derecho desde la Declaración Universal de los Derechos Humanos. Las normas fundamentales, tanto de la Unión Europea ${ }^{9}$ como de los países que la integran ${ }^{10}$, incorporan la igualdad y la no discriminación por razón de sexo como un derecho inalienable. Hay que añadir que su presencia en la cultura política se manifiesta, por ejemplo, en la polémica que suscitan declaraciones con sesgo sexista ${ }^{11}$. Esto no quiere decir que no persistan estereotipos discriminatorios (Valcárcel, 2004) que se filtran desde los chistes hasta las series de televisión, pero hay una asunción de la igualdad entre mujeres y hombres en el discurso oficial que deslegitima la expresión de actitudes machistas.

Respecto al entramado internacional, estaría formado por las influencias entre naciones y a través de los organismos internacionales. Aquí se reconoce el papel de

\footnotetext{
${ }^{6}$ En cambio, la influencia de los movimientos feministas en las actuaciones concretas de las instituciones, ha sido menor (Martínez, 2014: 158). Sobre la importancia del movimiento feminista en España durante los años 70, véase Astelarra (2005).

${ }^{7}$ En 1987, el Partido Socialista Obrero Español (PSOE) estableció una cuota de presencia femenina en las listas electorales del 25\%. Antes, en 1982, el Partit dels Socialistes de Catalunya (PSC) había introducido las cuotas al reservar a las mujeres el 12\% de los puestos en el partido y en las candidaturas electorales. Actualmente, la Ley Orgánica 3/2007, de 22 de marzo, para la igualdad efectiva de mujeres y hombres (LOI) marca el criterio de presencia equilibrada en una representación de ambos sexos no superior al 60\% ni inferior al 40\%. (LOI, 2007: Disposición adicional primera).

${ }^{8}$ Durante el gobierno del socialista José Luis Rodríguez Zapatero (2004-2011, VIII y IV legislaturas) las políticas de igualdad de género en España han tenido el mayor desarrollo legislativo. A nivel institucional, la creación del Ministerio de Igualdad fue un hito, aunque de corta duración (2008-2010).

${ }^{9}$ Carta de Derechos Fundamentales de la Unión Europea (2012: art. 23)

${ }^{10}$ Constitución Española (1978: art.14 y 9.2).

${ }^{11}$ En este sentido, fueron muy criticadas las declaraciones de Miguel Arias-Cañete, candidato del PP a las últimas elecciones europeas (2014) a raíz del debate mantenido con la candidata del PSOE, Elena Valenciano. Afirmó que era muy complicado debatir con una mujer porque "Si haces un abuso de superioridad intelectual, parece que eres un machista y estás acorralando a una mujer indefensa” Programa Espejo Público (Antena 3, 16.5.2014); “Arias Cañete dice que fue benévolo con Valenciano para no parecer machista”, El País, 16.5.2014. A fecha 13 de agosto de 2014, parece que estas declaraciones tenían un peso negativo en su futuro como comisario de la Unión Europea (véase el artículo "Rajoy busca el apoyo de Merkel para que Cañete logre algún cargo de comisario”. El País, 13.8.2014) sin embargo, la Comisión de 28 miembros, incluye 9 mujeres (32\%).
} 
Naciones Unidas y de la Unión Europea. Esta última ha tenido una importancia directa en el desarrollo nacional de las políticas de igualdad porque el estado español, como miembro de la UE, está condicionado por la normativa comunitaria ${ }^{12}$. Antes de pertenecer a la Comunidad Económica Europea (CEE), el gobierno de UCD participó en la década de la mujer de Naciones Unidas (1975-1985) y el Instituto de la Mujer vinculó la creación del primer plan de igualdad de oportunidades a las políticas europeas ${ }^{13}$. Se puede afirmar que los partidos fueron más permeables al contexto internacional que a las demandadas del feminismo militante español (Astelarra, 2005: 137-144).

Entonces, la forma en que se relacionen los distintos agentes del sistema político provoca variaciones en el sistema mismo. El doble movimiento de flujos entre la sociedad y el régimen político ha sido asimilado al sistema circulatorio sanguíneo: la sociedad demanda cambios al régimen político a través de sus actores y el régimen político elabora políticas públicas para satisfacer esa demanda (Alcántara, 1995: 57). Siguiendo con el tema de estudio, el feminismo como movimiento social y el feminismo institucional ${ }^{14}$ demandan cambios y los protagonizan no sólo en una retroalimentación con la sociedad, también con otros actores y organismos, así como con el resto de instituciones del estado. Esta retroalimentación que se produce en el sistema político afecta al régimen y a la sociedad. Se afirma desde el feminismo teórico que es necesario avanzar hacia democracias inclusivas que integren a otros colectivos, a otros públicos. La misma categoría de públicos se amplía: no se constituyen de una vez y para siempre, sino que un mismo individuo puede transitar de uno a otro (Young, 2000). De hecho, las sociedades son más plurales y se reconocen como tales. Este reconocimiento importa, porque el problema ha sido la falta de visibilidad de parte de la ciudadanía.

Las instituciones, como estructuras estables -en tanto que comúnmente aceptadas- formadas por las distintas organizaciones y los procesos que se generan, posibilitan esa interacción entre la ciudadanía y la esfera política. En palabras de Young (2011: 84), “organizan la acción como acción colectiva"15. El movimiento

${ }^{12}$ Cualquier estado europeo que quiera ser miembro de la Unión Europea, debe incorporar sus valores, entre los que está el de la igualdad entre mujeres y hombres (Tratado, 2012: art. 2); pero además, parte de la normativa europea tiene carácter vinculante (Tratado de Funcionamiento, 2012: art. 288)

${ }^{13}$ Primer Plan para la Igualdad de oportunidades de las Mujeres 1988-1990. España había entrado en la CEE en 1986.

${ }^{14}$ Por feminismo institucional se entiende el vinculado a organismos como el Instituto de la Mujer en España, ONU Mujeres, el Instituto Europeo para la Igualdad de Género, o las unidades de igualdad de las universidades. Abarcaría a las instituciones, a las personas que trabajan en ellas, los programas que desarrollan, así como su producción documental.

${ }^{15}$ Esta autora destaca la importancia de las instituciones a la hora de organizar la acción colectiva: es un tema de justicia, que tiene que ver con la responsabilidad individual, asumir la diferencia entre grupos en el ámbito público y político para, a partir de ahí, promover la participación de todos en las instituciones. Young sigue la propuesta de Mouffe, de un 
puede iniciarse en un sentido o en otro: hay veces que el régimen político se anticipa en la identificación y propuesta de resolución de un problema; otras veces es al revés. Por ejemplo, en España, la violencia de género entró en la agenda política a raíz de la aparición de mujeres maltratadas en la televisión ${ }^{16}$. Fue la presión de los medios de comunicación, que canalizaron la voz de estas mujeres como parte de la sociedad, la que caracterizó la violencia de género como un problema social y provocó un cambio político ${ }^{17}$. Sin embargo, otros cambios se han promovido desde instancias gubernamentales, sobre todo a partir de la promulgación de Ley Orgánica 3/2007, de 22 de marzo, para la igualdad efectiva de mujeres y hombres $(\mathrm{LOI})^{18} \mathrm{y}$ debido a su carácter integral. Sirvan como ejemplo las acciones positivas o el criterio de presencia equilibrada. La importancia de las instituciones se destaca porque aportan coordinación y continuidad a unas políticas que no pueden quedarse en acciones aisladas.

\subsection{LA IGUALDAD DE GÉNERO EN LA AGENDA PÚBLICA}

Se iniciaba este apartado con la definición de políticas públicas como "productos institucionalizados del sistema”. Así, sintetizando la exposición anterior, habría que entender éstas como acciones del gobierno que se hacen efectivas a través de la administración pública para dar respuesta a una demanda. Ésta habrá sido previamente identificada y definida como un problema a solucionar, en un contexto socio-político determinado por un sistema de valores. Entonces, para que un tema sea objeto de la actuación política ha tenido que ser (1) formulado como problema, (2) considerada viable su solución, (3) incluido en la agenda política. El promotor de su inclusión en la agenda puede ser (1) el propio gobierno, (2) la ciudadanía a través de sus actores o (3) la influencia internacional. El entramado institucional importa como soporte de la interrelación entre los actores que intervienen en el proceso. El contexto incluye la cultura política que expresan los actores y la influencia internacional.

pluralismo democrático radical, cuando propone una política de la diferencia que no esencialice lo distinto, sino que asuma una especificidad establecida en las relaciones entre grupos sociales y entre éstos y las instituciones. Los grupos se crean por afinidades entre personas, en base a la forma de vida o a determinadas prácticas. Tampoco los grupos son algo esencial: los individuos pueden pertenecer a distintos grupos sociales y variar sus adscripciones (Young, 2000).

${ }^{16}$ En 1997 Ana Orantes fue quemada viva por su marido. Días antes había contado en un programa de televisión su historia de maltrato. A partir de este momento, las denuncias aumentan y comienzan a contabilizarse las muertes. (Castillejo Manzanares: 2005)

${ }^{17}$ Los cambios han sido muchos: la diferenciación de este tipo de violencia respecto a otras producidas en el ámbito doméstico, la generación de estadísticas, la promulgación de una ley orgánica (Ley Orgánica 1/2004, de 28 de diciembre de Medidas de protección integral contra la violencia de género), la creación de observatorios de violencia de género, la redacción de protocolos de actuación para evitarla, etc..

${ }^{18}$ Considerada pionera en el entorno de la Unión Europea porque introduce el principio de igualdad de género en todas las estructuras de la sociedad. 
Para el caso de las políticas de igualdad entre mujeres y hombres, éstas deberían caracterizarse por a) ajustarse a una perspectiva: la de género; b) responder a una estrategia global: el gender mainstreaming; y c) una fuerte institucionalización que aporte coordinación al proceso y continuidad en el tiempo ${ }^{19}$. Su inclusión en la agenda política española ha sido promovida fundamentalmente por la influencia internacional, condicionada a su vez por las demandas del feminismo. Respecto a la heterogeneidad de los públicos afectados e instancias comprometidas, sus pretensiones podrían calificarse de universalistas: (1) aspiran a un cambio que afecte a toda la sociedad (hombres y mujeres de todas las edades), (2) su carácter integral exige proyección en diversos ámbitos (educativo, sanitario, económico, judicial, familiar, etc.), (3) el gender mainstreaming significa afectar a los distintos niveles de gobierno y de manera coordinada, así como a otras acciones, instituciones y actores, tengan o no relación directa con la igualdad.

A partir de aquí, se proyectan una serie de temas relevantes para desbrozar las causas de una desigualdad que persiste en nuestro entorno. Los análisis sobre la evolución de la igualdad en el marco de los derechos humanos, su relación con la gobernanza europea, así como su importante desarrollo legislativo en el empleo y el acceso masivo de las mujeres a la educación superior, construyen un marco favorable a la igualdad que contrasta con la realidad de los datos. Por esto es importante entender qué significa y qué aporta el traslado del concepto de género desde las teorías feministas a las políticas públicas como perspectiva.

\section{LA PERSPECTIVA DE GÉNERO O LA MIRADA DE LA SOSPECHA}

Asumir la perspectiva de género significa ir más allá de los problemas de las mujeres (Consejo de Europa, 1999; Lombardo, 2003b): es instaurar la mirada de la sospecha sobre una realidad desigual por razón de sexo. Cuando dicha perspectiva se traslada a las políticas públicas, se advierte el carácter estructural de la desigualdad. Ello es importante a la hora de decidir cómo intervenir desde los poderes públicos: va a condicionar la forma de hacerlo. Así, a partir de la Conferencia de Nairobi (1985) se propone la adopción de una nueva estrategia: el gender mainstreaming ${ }^{20}$ que, desde la Conferencia de Beijing (1995), significa actuar en todos los ámbitos, a todos los niveles y de manera coordinada, al tiempo que se pide su traslado a todos los gobiernos. El

\footnotetext{
19 “La institucionalización de las políticas públicas de género es el proceso mediante el cual una nueva práctica se incorpora a las instrucciones del Estado, se hace estable, reitera en el tiempo y por lo tanto permite su seguimiento y evaluación”. Astelarra (2005: 137).

${ }^{20}$ El término mainstreaming se origina en la década de 1980 en Gran Bretaña como el proceso de incorporación de la sensibilidad medioambiental, y significa "llevar a la corriente principal del desarrollo” estas políticas. De ahí pasa a la Tercer Conferencia Mundial sobre la Mujer de Naciones Unidas (Nairobi, 1985) como mainstreaming de género y entra en el lenguaje de la Unión Europea en 1991, incorporándose como estrategia de desarrollo comunitario (en Suecia un año antes). Casco (2003).
} 
problema de la desigualdad entre mujeres y hombres se perfila de forma distinta y las instancias políticas precisan adaptar la intervención (Alfama y Alonso, 2015).

Abordar la desigualdad desde la esfera pública de esta manera ha tenido un recorrido en el tiempo, pues el problema no se ha formulado igual ni de una forma única. En este momento, el concepto de género es central pero el propio concepto ha sido discutido y revisado ${ }^{21}$ (Tabla 1). Hay que reconocer que el debate feminista ha contribuido a fijar, a nivel internacional, un corpus teórico y práctico sobre qué es la desigualdad, qué consecuencias tiene para los individuos y cómo abordarla (¡Error! No se encuentra el origen de la referencia.). Así, su carácter multidimensional y la constatación de que las consecuencias son negativas para toda la sociedad, llevan a proponer un método transversal.

¿Qué implicaciones tiene incorporar la perspectiva de género a las políticas públicas? Significa reconocer que la desigualdad entre hombres y mujeres existe, que no debería existir y que tiene carácter estructural: las manifestaciones concretas, y toda la casuística, son muestras parciales de la desigualdad. El problema está en que el sistema social -con los subsistemas educativo, político, económico y legislativo, así como cultural- institucionaliza el sistema de género y esto influye de manera distinta en las oportunidades de mujeres y hombres ${ }^{22}$.

Así pues, asumir la perspectiva de género implica adoptar una posición proactiva y funcionalista del género. Es decir, en tanto en cuanto sirve para descubrir el carácter sistémico de la desigualdad en sociedades determinadas, a diferentes niveles y en distintas áreas, como la enseñanza y el trabajo. Por eso la estrategia proporcional a esta perspectiva es el gender mainstreaming. No es sólo un método de análisis de la realidad, sino también de corrección y prevención que debe guiar las políticas públicas. La centralidad del gender mainstreaming radica en que la falta de correspondencia y de coordinación entre acciones hace insuficiente la igualdad formal. Pero además, con la transversalidad se pretende integrar la perspectiva de género desde las etapas iniciales de los procesos hasta su evaluación ${ }^{23}$, de modo y manera que también influya en la asignación de recursos y en las metas ${ }^{24}$. Además de la coordinación no hay que olvidar

${ }^{21}$ Hawkesworth (1999: 4-6) recopila los usos del género, como concepto para rebatir el determinismo biológico, y las múltiples definiciones presentes en la literatura feminista desde la década de 1970 hasta inicios de 1990.

22 "Gender systems are institutionalized through education systems, political and economic systems, legislation, and culture and traditions. In utilizing a gender approach the focus is not on individual women and men but on the system which determines gender roles / responsibilities, access to and control over resources, and decision-making potentials.” Office of the Special Adviser on Gender Issues and the Advancement of Women (OSAGI, 2001b).

${ }^{23}$ Alfama y Alonso (2015: 27-33) describen las herramientas para desarrollar el gender mainstreaming.

24 "ECOSOC Agreed Conclusions 1997/2 provided a clear definition of the mainstreaming strategy as: ...the process of assesing the implitations for women and men of any planned action, including legislation, policies or programmes, in all areas and at all levels. It is a strategy for making women's as well as men's concerns and experiences an integral dimension of the 
la necesidad de acciones positivas y el mantenimiento de políticas sectoriales. Así, Naciones Unidas advierte de que la transversalización como estrategia no elimina los programas específicos de igualdad de género y se considera complementaria a las medidas que conducen al empoderamiento de las mujeres; tampoco vuelve irrelevantes a los especialistas, ni suple la necesidad de recursos diferenciados (Lombardo, 2003b).

\subsection{IDEOLOGÍA DE GÉNERO}

Uno de los réditos más importantes del concepto de género sería su traslado a la praxis política y la consecuente formulación de la llamada “ideología de género". En este artículo esto significa que la igualdad entre mujeres y hombres es el postulado necesario para construir sociedades justas y respetuosas con los derechos humanos. El sentido de este presupuesto se hace explícito a partir de la definición de "ideología” de la RAE ( ${ }^{a}$ acepción): "Conjunto de ideas fundamentales que caracteriza el pensamiento de una persona, colectividad o época, de un movimiento cultural, religioso o político, etc.”

Se admite que el concepto de género tiene carga ideológica porque refleja el pensamiento de una parte de la sociedad actual acerca de la posición pasada y presente de las mujeres, así como de su significado. Adoptar o no este concepto lleva a un posicionamiento distinto con relación a una realidad que es interpretada. Esto también implica hacer una valoración sobre lo que hay y lo que debe ser cambiado. Así, cuando se hacen expresos los postulados no se rehúye el debate con otras ideas.

El problema es que desde algunas posiciones políticas conservadoras se descalifica la "ideología de género" como "ideología”, es decir, se atribuye un matiz peyorativo a este término para trasladarlo al concepto de género. La pregunta es, ¿qué se quiere decir con esto cuando, según la definición que se aporta, es improbable que haya alguna acción política exenta de ideología? Si se tienen en cuenta los múltiples enfoques de lo que significan las ideas en el análisis de las políticas públicas, se podría afirmar que se utiliza un concepto marxista de "ideología": ideología como falsa creencia que se impone por los actores dominantes para mantener el control sobre los dominados (Harguindéguy, 2013: 140-142). Esto resulta chocante cuando precisamente la ideología de género es un ataque frontal a la ideología patriarcal que, ésta sí, subordina a las mujeres. Además, el feminismo que aquí se postula es una apuesta por la diversidad y la autonomía individual, de mujeres y hombres, en el marco de los derechos humanos.

En definitiva, la ideología de género como nuevo paradigma frente a la ideología patriarcal, es considerada desde el discurso conservador como algo negativo. En esta línea, hay una tendencia a reinterpretar el concepto de género, unas veces sustituyendo el significante, otras el significado. Así, en lugar de violencia de género, se habla de

design, implementation, monitoring and evaluation of policies and programmes in all political, economic and societal spheres so that women and men benefit equially and inequality is not perpetuated. The ultimate goal is to achieve gender equality.” OSAGI (2001a). 
violencia doméstica; o se caracteriza la violencia estructural como la que se ejerce contra el rol reproductivo de las mujeres ${ }^{25}$. Esta tergiversación no es sólo una cuestión de lenguaje: en el contexto de la crisis económica va acompañada de cambios institucionales, legislativos y de reducción de instrumentos destinados a implementar la igualdad de género. Esta situación ha llevado a Paleo y Alonso (2014; Alonso, 2015) a considerar la crisis como una ventana de oportunidad para reducir las políticas de igualdad de género que es utilizada, mayormente, por los partidos políticos conservadores. Las consecuencias fundamentales han sido (véase también Lombardo y León, 2014): recortes presupuestarios en base a la austeridad, eliminación o pérdida de rango de organismos e instituciones con el argumento de la racionalización administrativa y cambios legislativos que promueven el rol cuidador y dependiente de la mujer (por ejemplo con la modificación de la ley del aborto y el destino de fondos a redes y campañas pro-vida).

Por el contrario, las políticas públicas de igualdad con perspectiva de género se caracterizan por promover un proyecto social común, que integre a hombres y mujeres sin condicionar sus oportunidades por atribuirles un rol concreto. Dicho proyecto se fundamenta en reconocer que la mujer es postergada, que los universales son excluyentes, que no hay neutralidad y que la igualdad formal es insuficiente.

\section{TESIS FUNDAMENTALES}

Como se ha dicho poco antes, la tesis principal es que el concepto de género es clave para entender el desarrollo de las políticas públicas de igualdad desde la década de 1990 y que dicho desarrollo va ligado a la adopción de la perspectiva de género, necesaria para (1) entender los mecanismos por los cuales la mujer ha sido preterida históricamente; (2) desvelar que los universales son excluyentes porque responden a un modelo patriarcal de representación que subsume lo diferente, bajo la apariencia de generalidad, para eliminarlo; (3) desmontar la supuesta neutralidad de la realidad social y de las instituciones; (4) denunciar que la igualdad formal alcanzada en las sociedades democráticas occidentales no es una igualdad real $\mathrm{y}$, por tanto, aunque necesaria es insuficiente. La perspectiva de género, en un doble movimiento de denuncia y corrección, es un posicionamiento ideológico.

(1) La mujer postergada. La realidad social aún está conformada desde parámetros que deben ser revisados porque dificultan la emancipación de las mujeres ${ }^{26}$. El

${ }^{25}$ Así Ruiz-Gallardón (Ministro de Justicia de España, 22/12/2011 - 23/09/2014) , cuando dice que la violencia estructural se ejerce contra la mujer cuando se la impide ser madre, que es su verdadera vocación "la libertad de la maternidad es la que hace a las mujeres auténticamente mujeres". El País, 27 de marzo de 2012.)

${ }^{26}$ Kate Millet en Sexual Politics (1970; edición en español utilizada: 1995) explica como las minorías raciales y las mujeres no tienen posibilidad de organizarse porque son excluidas de las instituciones en el marco del sistema patriarcal. El denominador común de los distintos sistemas patriarcales es la relación de poder entre los sexos. Una estrategia del opresor consiste en 
concepto de género va más allá de lo que aporta la crítica del "patriarcado" por desmontar el fundamento biologicista que subyace a la construcción histórica de "la mujer". Esta construcción forma parte de nuestra cultura y persiste al ser la base de un discurso normativo acerca de lo que significa "ser hombre" y "ser mujer". A partir del mismo no sólo se establece una jerarquía entre "los dos sexos", sino que también se excluye como "perversión” o "juego" opciones que no estén dentro de la heterosexualidad (Rubin, 1986). Este discurso normativo, más o menos explícito, más o menos simbólico, es como la argamasa del sistema sexo-género que lleva a postergar a la mujer.

El género, como herramienta conceptual, cuando se traslada a las políticas públicas obliga a revisar aquello que indique persistencia de desigualdades y discriminación. Visibilizar a las mujeres requiere acciones que van desde la más sencilla de desagregar los datos por sexo, hasta destacar su papel en la historia o en la ciencia. Ofrecer modelos de referencia tiene además un carácter didáctico en la superación de estereotipos. Otras propuestas se articulan en la demanda de que se valore económicamente el trabajo desempeñado tradicionalmente por las mujeres bajo el concepto de "cuenta satélite de hogares" ${ }^{27}$. Se recurre así al mismo paradigma economicista, que penaliza el rol cuidador, para contabilizar monetariamente un trabajo no mercantil. Esto implica reconocer que tiene repercusiones en la economía, aunque el diseño del modelo tenga dificultades ${ }^{28}$.

Por último, el imperativo de posicionar a la mujer en la esfera pública se sintetiza en el concepto de "empoderamiento": es la alternativa a su reclusión tradicional en el

atribuir a las mujeres determinadas cualidades relacionadas con su posición subordinada. De ahí la baja autoestima y que llegue a asumir los valores patriarcales y los transmita. De esta forma, el patriarcado se apoya, además de en la violencia, en el consenso, siendo la familia la que reproduce los roles diferenciados. No obstante, y aunque Millet reconoce que la discriminación patriarcal está tan arraigada socialmente que no puede distinguirse la parte natural de la cultural, también sostiene que hay posibilidad de cambiar esto: desde el discurso crítico de las primeras feministas se han generado reformas.

${ }^{27}$ Conferencia de Beijing (1995). En España, en 1998, se aprobó en el Congreso una propuesta no de ley para medir ese trabajo no remunerado, sin embargo la iniciativa se paralizó por falta de asignación económica (Nuño, 2010: 179). Actualmente es la Encuesta de Empleo del Tiempo la que pretende estimar este valor. Lo que se quiere destacar aquí es lo que estas propuestas tienen de reconocimiento.

${ }^{28}$ Casero y Angulo (2008) señalan que la incorporación de estas cifras al PIB reduciría las diferencias entre países desarrollados y países en desarrollo. En el caso de los primeros, al estar externalizada la atención a dependientes, se crean servicios que computan en la economía nacional porque generan valor añadido y crean puestos de trabajo; mientras que en los segundos se asumen principalmente por las mujeres. Destacan así sus posibilidades como herramienta de análisis económico y social. Sin embargo, aunque hay un manual de Eurostat sobre la materia, también indican los problemas al no haber criterios homogéneos a nivel internacional para estructurar la recogida de información, realizar los cálculos, etc. El diseño de la propia Encuesta de Empleo del Tiempo, según los mismos autores, no se adecuaría a otros modelos, por lo que no se pueden establecer comparaciones. 
espacio doméstico y el camino para alcanzar la autonomía mediante su participación en la toma de decisiones ${ }^{29}$.

(2) El universal falaz. El universal, al hacer creer a todos los individuos que el sistema formal los incluye, contribuye con el discurso normativo a consolidar la dualidad de género. La trampa consiste en que, bajo la apariencia de universalidad, se refleja la situación del grupo hegemónico y se excluye a "los otros", como marginales o diferentes. Ocurrió en la revolución francesa, cuando Olimpia de Gouges escribió la Declaración de los derechos de la mujer y de la ciudadana (1791) porque la Declaración de los derechos del hombre y del ciudadano olvidó a las mujeresciudadanas; también las convenciones y declaraciones de Naciones Unidas posteriores a la Declaración Universal de los Derechos Humanos (redactados inicialmente como "del hombre"), han tenido que insistir en los derechos de la mujer ${ }^{30}$ como derechos humanos.

No tener en cuenta a los otros en su especificidad de grupo, es algo que no ocurre sólo con las mujeres sino también con otros colectivos. Así por ejemplo, los homosexuales, mientras no hagan explícita su condición, tendrán todos los derechos civiles. Pero cuando una persona quiere contraer matrimonio con otra de su mismo sexo, la falta de una ley que lo permita la excluirá, por opción sexual, de derechos asociados a la familia que van desde permisos familiares hasta la fiscalidad ${ }^{31}$. Un ejemplo de esta limitación de derechos es el caso de las adopciones ${ }^{32}$.

La perspectiva de género demanda a los poderes públicos arbitrar las condiciones que posibiliten la integración de una diversidad de públicos (Fraser, 1993), del “otro-

${ }^{29}$ Ello requiere su presencia en los órganos de representación, gobierno y toma de decisiones a nivel local, regional, nacional e internacional. La Plataforma de Acción basa la consecución de la igualdad y el adelanto de la mujer en la interrelación entre todos los objetivos estratégicos, si bien es destacable el objetivo G: "La mujer en el ejercicio del poder y la adopción de decisiones" porque pone de relieve la importancia de que la mujer participe activamente en dos fases del ciclo de políticas públicas: la toma de decisiones y la implementación. Naciones Unidas (Beijing, 1995: 97-104).

${ }^{30}$ La Comisión de la Condición Jurídica y Social de la Mujer (CSW), desde su creación en 1946, ha concretado su labor en redactar normativa específica sobre los derechos de la mujer. Es significativo que la Convención sobre la eliminación de todas las formas de discriminación contra la mujer de 1979 se considere la carta internacional de los derechos humanos de las mujeres. También la Declaración de Beijing (1995), recuerda en el párrafo 14: "Los derechos de la mujer son derechos humanos"

${ }^{31}$ Es otra la discusión acerca de si estos derechos "familiaristas" favorecen o no la igualdad de género y las propuestas en la línea de adoptar medidas universales que afecten a los individuos, no a su posible condición de cuidadores (Pazos, 2013). Ahora lo que se demuestra es que la opción sexual limita las oportunidades en un momento determinado.

${ }^{32}$ A raíz de una sentencia del Tribunal Supremo ruso de agosto de 2013, se han paralizado las adopciones en países que permiten el matrimonio homosexual, como es el caso de España, hasta que no se firme un acuerdo bilateral que garantice que los niños adoptados no serán criados por homosexuales. El gobierno español ha consentido (El País, 20/12/2013). 
concreto" (Benhabib, 2006). Tenerlos en cuenta demanda un concepto extenso e inclusivo de ciudadanía que puede vincularse con el concepto de gobernanza “ampliada” definido por la Unión Europea ${ }^{33}$.

(3) No hay imparcialidad. La realidad social y las instituciones no son neutrales: condicionan desde el origen, y de forma distinta, la vida de los hombres y de las mujeres. La desigualdad es un problema de justicia que se inicia en la familia como primer agente de socialización (Okin, 1996a, 1996b ) y que, en el ámbito público, requiere ir más allá del paradigma distributivo (Young, 2000). Se reproducen estos dos modelos asimétricos de relación como si fueran igualitarios, hasta el punto de que las críticas se subsanan con el subterfugio de la complementariedad. El discurso sobre la no discriminación por razón de sexo, presente en la cultura occidental como un principio que la informa desde la Declaración Universal de los Derechos Humanos (1948), también contribuye a crear la impresión de que el marco es imparcial, mientras los usos sociales son otros. Por eso hace falta un distanciamiento crítico, en perspectiva de género. Por ejemplo, los análisis sobre el empleo y la enseñanza universitaria ${ }^{34}$, demuestran cómo la persistencia de estereotipos culturales contribuye a fijar el rol cuidador de las mujeres ${ }^{35}$. Esto es así hasta el punto de que las medidas que el mercado

${ }^{33}$ El buen gobierno requiere aumentar el compromiso ciudadano en un sistema político comprensible, cercano y eficaz: participación y transparencia son claves para incrementar la legitimidad de las políticas e instituciones de la Unión. Por el contrario, los retrocesos en igualdad de género significarían un déficit democrático al excluir a parte de la población de la esfera política. La gobernanza, frente a este riesgo, ofrece la posibilidad de introducir un elemento normativo al reclamar mayor intervención ciudadana. Es un modo de reforzar los valores que sustentan la Unión Europea -entre ellos la igualdad- por encima de los intereses meramente mercantilistas. En esta línea, se entiende que la legitimidad de la Unión depende de una ciudadanía comprometida y activa en los distintos niveles del proceso político, desde la formulación de políticas públicas hasta su ejecución (mayor participación cuantitativa y cualitativa).

${ }^{34}$ La persistencia de la brecha salarial de género (en España, en 2014, era del 22,9\% según el Plan Estratégico de Igualdad de Oportunidades 2014-2016); la escasa presencia de mujeres en puestos directivos; el bajo número de catedráticas universitarias (curso 2011-2012: no llegan al 20 \% en España, según datos del Instituto de la Mujer), etc., indican que, mientras el discurso oficial se construye como igualitario, la situación de las mujeres es de desventaja. La promoción de su rol cuidador desde distintas instancias (familia, medios de comunicación, etc.) las hace posponer su desempeño profesional al tiempo que se produce una suerte de discriminación estadística.

${ }^{35}$ La Comisión de Empleo y Asuntos Sociales del Parlamento Europeo (Informe FEEM, 2012), señala que las diferencias salariales entre hombres y mujeres se deben principalmente a "los estereotipos y la percepción de la mujer solo a través del prisma de la división tradicional de roles”. Véanse, en el mismo informe, las recomendaciones a la Comisión de Derechos de la Mujer e Igualdad de Género. 
de trabajo prevé para "conciliar”, o determinadas ventajas fiscales ${ }^{36}$, no hacen sino anclar a las mujeres en su papel de cuidadoras. Las consecuencias afectan a las oportunidades y a la calidad de vida.

(4) No es suficiente la igualdad formal para cambiar la realidad social y las instituciones que la soportan. Pensar que la legislación es suficiente está vinculado a la falacia de que hay imparcialidad. Pero la persistencia de desigualdades de género y el retroceso en políticas públicas, desmonta esta suficiencia. Por ejemplo, si se tiene en cuenta la situación de las mujeres en la educación, en España, desde los años 70 ha ido en aumento su ingreso en la universidad: su acceso a la educación superior en igualdad de condiciones está garantizado por ley ${ }^{37}$. Sin embargo, persiste una diferencia significativa entre el número de mujeres que cursan estudios en titulaciones del área de Ciencias de la Salud y de Humanidades respecto a las titulaciones técnicas ${ }^{38}$. Tampoco evoluciona igual la carrera profesional dentro de la misma universidad, detectándose sesgos verticales de género ${ }^{39}$. Se podrá argumentar que hay un traslado de los roles familiares a la educación, que faltan referentes femeninos en ciertas áreas, que las hijas aún reproducen patrones aprendidos de sus madres, etc., pero sensu stricto, no hay ninguna ley que impida a una mujer matricularse en una titulación de ingeniería naval u optar a cátedra, y la promoción profesional en la institución se basa en el mérito y la capacidad.

\section{ALGUNAS CONSECUENCIAS}

Todo esto lleva a plantear las consecuencias de integrar la perspectiva de género en las políticas públicas. La primera sería que la ya cuestionada frontera entre lo

${ }^{36}$ Tributación conjunta, pensiones de viudedad, reconocimiento del tiempo cotizado en las excedencias vinculadas al cuidado o las prestaciones por cuidado en el entorno familiar (Pazos, 2013).

${ }^{37}$ La Ley 14/1970, de 4 de agosto, General de Educación y Financiamiento de la Reforma Educativa, unificó todo el sistema educativo y consiguió la plena escolarización de todos los españoles durante los ocho cursos obligatorios y gratuitos de la Enseñanza General Básica (EGB). También estableció la gratuidad de la enseñanza en el Bachillerato y en la Formación Profesional, la educación mixta y un sistema de becas para promover la igualdad de oportunidades.

${ }^{38}$ Mujeres estudiantes en el área de Ciencias de la Salud: 73\%; hombres estudiantes en Ingeniería y Arquitectura: 72\% (Universidades públicas españolas, curso 2010-2011. Michavila, 2014).

${ }^{39}$ En la universidad española, donde las alumnas superan el 50\%, el punto de inflexión se produce a partir del doctorado: en el Programa Nacional de Contratación e Incorporación de Recursos Humanos. En 2012, las mujeres contratadas en el programa Ramón y Cajal fueron el 39 \% y en el Juan de la Cierva, el 38,2 \% A partir de aquí, las profesoras universitarias no llegan al 40 \% (Fuente: Plan Nacional de I+D+i, convocatoria 2012. Los datos están actualizados en noviembre de 2013. Ministerio de Educación, Cultura y Deporte, 2014: 116127). 
público y lo privado perdería rigidez; en segundo lugar, la incidencia de las desigualdades en las oportunidades de los individuos se cuestiona como un tema de justicia; en tercer lugar, el concepto de ciudadanía se amplía ${ }^{40}$.

La pregunta es qué aporta y qué exige adoptar la perspectiva de género, puesto que la discriminación de la mujer y su falta de derechos ya habían sido denunciadas. La respuesta es la necesidad de una visión crítica y de conjunto. Por eso la división tradicional de ámbitos privados y ámbitos públicos no puede escapar a este enfoque, pues la familia, el trabajo entendido en el sentido amplio de profesión, y la ciudadanía están lastrados por la desigualdad. Pero además, hay una interrelación entre los distintos roles que cada individuo representa que ha afianzado la pérdida de valor del modelo social atribuido a las mujeres. Ello se concreta en: (1) la falta de reconocimiento de la incidencia del trabajo reproductivo sobre el trabajo productivo; (2) la contribución de la familia a la creación de los modelos de identidad social; (3) el menor desarrollo para las mujeres de los derechos de ciudadanía (Bustelo y Lombardo, ca. 2005: 7).

(1) La falta de reconocimiento de la conexión entre trabajo reproductivo y trabajo productivo ha tenido a su vez tres consecuencias: se ha restado importancia a la función de la familia como soporte emocional y de servicios básicos necesarios para el desarrollo del trabajo productivo (Nuño, 2010; Murillo, 2006); ha provocado su infravaloración como trabajo en sí y como aporte a la economía productiva (Durán, 2012); ha supuesto un freno a la incorporación de la mujer al mercado laboral en igualdad de condiciones. Abundando en lo anterior, a partir de la crisis de 2007, la empleabilidad de las mujeres no ha evitado que se hayan visto más afectadas por los recortes en dependencia o ayudas sociales (Lombardo y León, 2014). Por tanto, ni el hombre ni el estado han contribuido a "liberar" a la mujer de las tareas del cuidado. En

${ }^{40}$ Fraser y Gordon (1992), proponen revisar un concepto de ciudadanía vinculado en los Estados Unidos con los derechos civiles, de modo y manera que los derechos sociales están excluidos y, con ellos, quienes no tienen acceso a los primeros: mayoritariamente mujeres. La ciudadanía civil sigue el modelo del contrato, que presupone individuos con personalidad legal e independencia económica, mientras que lo social no tendría carácter contractual, sino que se asocia con caridad como algo que se ejerce de manera unilateral y voluntaria, sin reciprocidad, por lo que la persona beneficiada carecería de derechos. La oposición ideológica entre contrato y caridad, tiene para estas autoras una marca de género que desprotege a las mujeres al ser, en mayor número, las aspirantes a los derechos sociales. En Europa, al menos antes de la crisis iniciada en 2008, el modelo del estado de bienestar -sin entrar en diferencias entre países- se articula desde unos parámetros distintos al modelo estadounidense: el asistencialismo europeo tiene más que ver con la redistribución que con la caridad (Alcántara, 1995: 163-164). No obstante, puede ser interesante tener en cuenta el análisis de Fraser y Gordon en este momento de recortes sociales y, en cualquier caso, es válido a la hora de comparar la diferencia de derechos a los que da lugar un trabajo según sea o no productivo con relación, por ejemplo, a las pensiones contributivas en el primer caso y no contributivas en el segundo (Cobo, 2000; Pazos, 2013). 
resumen: ausencia de corresponsabilidad y fallo en la conciliación, respectivamente ${ }^{41}$.

(2) A partir de los papeles asignados por la familia tradicional, se ha creado un modelo de identidad femenina secundario respecto al de identidad masculina. Esto se traslada a la vida social. El problema añadido es la exclusión de las identidades no normativas que, aunque no es un tema central en este artículo, comparten con las mujeres la categoría de colectivo no hegemónico. La repercusión es que los modelos incorporan distintos valores y los asociados al modelo femenino son más restrictivos. Por eso inciden en las oportunidades de las mujeres y en su calidad de vida ${ }^{42}$.

(3) La división del trabajo y la organización de la vida privada condicionan a su vez los derechos de ciudadanía de las mujeres porque limitan su participación activa en el ámbito político, donde también son más vulnerables. Esta vulnerabilidad se manifiesta en fenómenos múltiples, desde el escrutinio de su físico, en base a unos estándares de tallas y vestuario, hasta la violencia sexual.

En definitiva, la perspectiva de género es una herramienta necesaria a la hora de diseñar políticas públicas en materia de igualdad. La corresponsabilidad es actualmente un horizonte que permitirá el avance hacia la igualdad material en sociedades donde los individuos sean indistintamente proveedores y sustentadores (Pazos, 2013). De acuerdo con esto, establecer sistemas más igualitarios se convierte en un postulado ético y crítico que se debe trasladar a los poderes públicos y, desde ahí, a la sociedad. La razón es que la igualdad real incide en las oportunidades, en la calidad de vida, y por eso afecta a todos los ámbitos, sin diferenciar públicos o privados. Al igual que los modelos de familia son cambiantes, desempeñar un rol u otro, cualquiera que sea, no tiene por qué vincularse al sexo, menos penalizarse.

\section{CONCLUSIONES}

En base a la exposición anterior, se puede concluir que la perspectiva de género en políticas públicas importa en el avance hacia la igualdad real. El debate feminista sobre las limitaciones del concepto de género, no invalida su funcionalidad como perspectiva. De hecho, la generalización de esta perspectiva como gender mainstreaming, se vincula a la posibilidad de establecer un nuevo paradigma que promueva mayor justicia social.

41 El concepto “conciliación” vincula como relación causa-efecto: mujer trabajadora desatención a la familia - problema sociolaboral. Sin embargo, la "corresponsabilidad" tiene en cuenta el reparto de las tareas del cuidado en la familia, no sólo la incidencia de éstas en el trabajo.

42 "No importa cuán formalmente iguales sean las mujeres, puesto que mientras sigan teniendo una responsabilidad desproporcionada respecto de las tareas domésticas, la crianza de los hijos/as y el cuidado de las personas enfermas y ancianas, y mientras su trabajo siga siendo algo privado, infravalorado, no remunerado o escasamente remunerado, el principio anticastas seguirá siendo violado y las mujeres estarán sistemáticamente en una situación de desventaja" (Okin, 1996a: 146) 
No obstante, hay que tener en cuenta que este desarrollo teórico de la perspectiva de género y su traslado a las políticas reglamentarias e institucionales, no ha calado suficientemente ni en la sociedad ni en los poderes públicos. Muestra de ello son los retrocesos habidos a partir de la crisis de 2007. De ahí la importancia de restablecer el marco teórico que ha propiciado el mayor avance en igualdad hasta la fecha y la necesidad de sistematizar los aportes de la perspectiva de género en políticas públicas.

En esta línea, se insiste en la necesidad de implementar políticas sectoriales y transversales de igualdad de género a partir del fortalecimiento institucional, la participación de los agentes sociales y el conocimiento experto. Se requiere de la implicación de los poderes públicos porque el marco es una democracia ampliada, lo cual significa reconocer la diversidad e incrementar la participación, lo que en nuestro entorno se vincula con la gobernanza europea.

Para avanzar hacia la igualdad real también hay que tener en cuenta los desajustes y las contradicciones entre las exigencias del mercado laboral y lo que la familia y la sociedad esperan de las mujeres, de su tradicional rol cuidador. Con relación a esto, hay que insistir en que no puede haber igualdad mientras falte reciprocidad: la mujer conquista posiciones tradicionalmente masculinas, pero el hombre no es tan proactivo en ocupar posiciones tradicionalmente femeninas. La pregunta es ¿qué justificaría el interés por un espacio que social y laboralmente ofrece desventajas?

De ahí que se postule la necesidad de desvincular a hombres y mujeres de los tradicionales roles de género. Para ello, además de fomentar el empleo femenino, hay que tener en cuenta la imbricación de las políticas sociales y las de igualdad de género, pues mientras no se solucionen los problemas que se vinculan al cuidado será difícil el intercambio de roles.

En definitiva, aunque la igualdad en políticas públicas ha ido ganando en concreción conceptual y legislativa ${ }^{43}$, no sólo no se ha alcanzado la igualdad real, sino que su falta de consolidación ha permitido retrocesos. En este sentido, la desigualdad en el terreno laboral puede calificarse de paradigmática, precisamente porque ha tenido un importante desarrollo legislativo en el contexto de la Unión Europea. La falta de equidad aquí, apunta a otros ámbitos y al carácter multicausal de la desigualdad de género. Por eso la mirada crítica que instaura la perspectiva de género, permitirá formular distintas propuestas.

\section{REFERENCIAS BIBLIOGRÁFICAS}

ALCÁNTARA SÁEZ, Manuel (1995): Gobernabilidad, crisis y cambio. Elementos para el estudio de la gobernabilidad de los sistemas políticos en época de crisis y cambio. México: Fondo de Cultura Económica.

ALCOFF, L. (2002): "Feminismo cultural vs. post-estructuralismo: la crisis de identidad de la teoría feminista”. Debats, 76, p. [1-26]

${ }^{43}$ En la Unión Europea y en España se ha pasado de la igualdad como un derecho (políticas simbólicas) a la igualdad legal (políticas reglamentarias). 
ALFAMA, Eva y ALONSO, Alba (2015). "Las políticas de género en la administración pública. Una introducción”. Revista Vasca de Gestión de Personas y Organizaciones Públicas, núm. 8, p. 24-41.

ALONSO, Alba (2015). "Las políticas de género en España: retrocesos y resistencias en tiempos de austeridad”. Ex aequo, $\mathrm{n}^{\circ}$ 32, p. 33-48.

ASTELARRA, Judith (2005): Veinte años de políticas de igualdad. Madrid: Ediciones Cátedra; Universitat de València; Instituto de la Mujer.

BENHABIB, Seyla (1992): "Una revisión del debate sobre las mujeres y la teoría moral”. Isegoría, vol. 5, p. 37-63.

BENHABIB, Seyla (2006): El Ser y el Otro en la ética contemporánea. Feminismo, comunitarismo y posmodernismo. Barcelona: Gedisa.

BORDIEU, Pierre (2007): La dominación masculina. $5^{\circ}$ ed. Barcelona : Editorial Anagrama.

CONSEJO DE EUROPA (1999): "Mainstreaming de género: marco conceptual, metodología y presentación de "buenas prácticas": informe final de las actividades del Grupo de Especialistas en Mainstreaming (EG-S-MS)”, Madrid: Instituto de la Mujer.

BUSTELO, María y LOMBARDO, Emanuela (ca. 2005): “Mainstreaming de género y análisis de los diferentes 'marcos interpretativos' de las políticas de igualdad en Europa: el proyecto MAGEEP”. Disponible en: http://pendientedemigracion.ucm.es/info/mageeq/documentos/Art ProyectoMageeq .pdf [actualizado: Julio, 8, 2014].

BUTLER, Judith (2001): El género en disputa: el feminismo y la subversión de la identidad. México: Paidós.

CASCO, Beatriz (coord.) (2003): "Mainstreaming. Una aproximación teórica”, Madrid: Instituto de la Mujer.

CASERO, Víctor y ANGULO, Carlos. (2008): "Una cuenta satélite de los hogares en España. 2003. Resultados derivados de la Encuesta de Empleo del Tiempo 20022003”. Madrid: Instituto Nacional de Estadística.

COBO, Rosa. (2000): Género. En, Amorós, C. (ed.) (2000 b): 10 Palabras clave sobre mujer, p. 55-83. Navarra: Editorial Verbo Divino.

COMISIÓN EUROPEA (2001): La gobernanza europea. Un libro blanco, DO C 287 de 12.12.2001, $\operatorname{COM}(2001) 428$ final.

DE BEAUVOIR, Simone. (2005): El segundo sexo. Madrid: Cátedra.

DE GOUGES, Olympe (1791): Declaración de los derechos de la mujer y de la ciudadana Disponible en: http://clio.rediris.es/n31/derechosmujer.pdf [actualizado: Julio, 12 2014]. 
DURÁN HERAS, Ma Ángeles. (2012): El trabajo no remunerado en la economía global. Bilbao: Fundación BBVA.

FRASER, Nancy y GORDON, Linda (1992): “Contrato 'versus' caridad: una reconsideración de la relación entre ciudadanía civil y ciudadanía social”. Isegoría 6, p. 65-82.

FRASER, Nancy (1993): "Repensar el ámbito público: una contribución a la crítica de la democracia realmente existente”. Debate feminista, marzo, 1993, p. 23-58.

FRIEDAN, B. (2009): La mística de la feminidad. $1^{\text {a }}$ ed. Madrid: Cátedra.

HARGUINDÉGUY, Jean Baptiste (2013): Análisis de políticas públicas. Madrid: Editorial Tecnos.

HAWKESWORTH, Mary (1999): “Confundir el género (Confounding gender)”. Debate Feminista, vol. 20, (octubre 1999), p. 3-48.

INFORME FEEM [de la Comisión de Derechos de la Mujer e Igualdad de Género del Parlamento Europeo], de 10 de mayo de 2012 (2011/2285(INI)). Bauer, E. (ponente). Parlamento Europeo. A7-0160/2012. Disponible en:

http://www.europarl.europa.eu/meetdocs/2009 2014/documents/juri/pr/878/878519 /878519es.pdf [actualizado: Julio, 19, 2014].

GILLIGAN, C. (2006): “Con otra voz: las concepciones femeninas del yo y de la moralidad”. En, López de la Vieja, M.T. et. al. (eds.). Bioética y feminismo, p. 1555. Salamanca: Ediciones Universidad de Salamanca.

GOBIERNO DE ESPAÑA. Ministerio de Educación, Cultura y Deporte (2014): “Datos y cifras del Sistema Universitario Español. Curso 2013-2014”. Disponible en: $\quad$ https://www.mecd.gob.es/dms/mecd/educacion-mecd/areaseducacion/universidades/estadisticas-informes/datoscifras/DATOS_CIFRAS_13_14.pdf [actualizado: Marzo, 14, 2014].

LOMBARDO, Emanuela (2003a): "La europeización de la política española de igualdad de género”. Revista Española de Ciencia Política, núm. 9, octubre 2003, p. 65-82.

LOMBARDO, Emanuela (2003b): "El mainstreaming de género en la Unión Europea". Aequalitas. Revista Jurídica de Igualdad de Oportunidades entre Mujeres y Hombres, vol. 10-15, mayo-diciembre 2003, p. 6-11.

LOMBARDO, Emanuela y LEÓN, Margarita (2014): "Políticas de igualdad de género y sociales en España: origen, desarrollo y desmantelamiento en un contexto de crisis económica”. Investigaciones Feministas, vol. 5, 13-35.

MARTÍN, Sara (2015). "Concepto de género: de las teorías feministas a las políticas públicas. La universidad pública española como estudio de caso”. Tesis doctoral dirigida por $\mathrm{M}^{\mathrm{a}}$ Teresa López de la Vieja. Universidad de Salamanca.

MARTÍNEZ, Eva (2014). Las formas de acción colectiva: partidos políticos y movimientos sociales. En, Lois, M. y Alonso, A. (coords). Ciencia política con perspectiva de género, p. 129-159. Madrid: Akal. 
MICHAVILA, Francisco (dir.). (2014). "La Universidad española en cifras 2012”. [Madrid]: Conferencia de Rectores de las Universidades Españolas (CRUE). Disponible en:

http://www.crue.org/Publicaciones/Documents/UEC/LA_UNIVERSIDAD_ESPAN OLA_EN_CIFRAS.pdf [actualizado: Marzo, 14, 2014].

MILLET, Kate (1995): Política sexual. Madrid: Cátedra, Universitat de València, Instituto de la Mujer.

MURILLO, Soledad (2006): El mito de la vida privada. De la entrega al tiempo propio. $2^{\mathrm{a}}$ ed. Madrid: Siglo XXI.

MOUFFE, Chantal (1993): "Feminismo, ciudadanía y política democrática radical”, Debate feminista, marzo, 1993, p. 3-22.

NACIONES UNIDAS. CEDAW (1979): “Convención sobre la eliminación de todas las formas de discriminación contra la mujer".

NACIONES UNIDAS (1995a): “Declaración y Plataforma de Acción de Beijing”. IV Conferencia Mundial sobre la Mujer, Beijing, del 4 al 15 de septiembre de 1995. Disponible en: http://www.un.org/womenwatch/daw/beijing/pdf/BDPfA\%20S.pdf [actualizado: Julio, 20, 2013].

NACIONES UNIDAS (1995b): "Informe de la cuarta conferencia mundial sobre la mujer: Anexos I-IV”. A/CONF.177/20/add.1. Disponibles en: http://www.un.org/esa/documents/ga/conf177/aconf177-20add1sp.htm [actualizado: Julio, 20, 2013]

NUÑO, Laura (2010): El mito del varón sustentador. Orígenes y consecuencias de la división sexual del trabajo. Barcelona: Icaria.

OKIN, Susan Moller. (1996a). Liberalismo político, justicia y género. En, Castells, C. (comp.). Perspectivas feministas en teoría política, p. 127-147. Barcelona: Paidós.

OKIN, Susan Moller. ( (1996b). Desigualdad de género y diferencias culturales. En, Castells, C. (comp.). Perspectivas feministas en teoría política, p. 185-206. Barcelona: Paidós.

OSAGI (Office of the Special Adviser on Gender Issues and the Advancement of Women). (2001a): "The development of the gender mainstreaming strategy". (CH rev. August 2001) .

OSAGI (2001b) "Important concepts underlying gender mainstreaming.”

Disponible en: http://www.un.org/womenwatch/osagi/pdf/factsheet3.pdf [actualizado: Julio, 21, 2014].

PATEMAN, Carole (1995): El contrato sexual. Barcelona: Editorial Anthropos; México: Universidad Autónoma Metropolitana.

PALEO, Natalia y ALONSO, Alba (2015). “¿Es solo una cuestión de austeridad? Crisis económica y políticas de género en España”. Investigaciones Feministas, vol. 5, 36-68. 
PAZOS, María (2013): Desiguales por ley: las políticas públicas contra la igualdad de género. Madrid: Los libros de la CATARATA.

RUBIN, Gayle (1986): "El tráfico de mujeres: notas sobre la economía política del sexo”. Nueva Antropología, vol. VIII, no 30, México, p. 95-145. Disponible en: http://www.juridicas.unam.mx/publica/librev/rev/nuant/cont/30/cnt/cnt7.pdf [actualizado: Julio, 9, 2014].

RUBIN, Gayle (1989): Reflexionando sobre el sexo: notas para una teoría radical de la sexualidad. En, Vance, C. S. (comp.). "Placer y peligro. Explorando la sexualidad femenina”, p. 113-190. Madrid: Revolución. Disponible en: http://webs.uvigo.es/xenero/profesorado/beatriz_suarez/rubin.pdf [actualizado: Julio, 9, 2014].

SCOTT, Joan (1986): “Gender: A Useful Category of Historical Analysis". The American Historical Review, vol. 91, n 5, p. 1053-1075; versión traducida: "El género: una categoría útil para el análisis histórico”. En, Lamas, M. (comp.) (1996). El género: la construcción cultural de la diferencia sexual, p. 265-302. México: PUEG.

UNIÓN EUROPEA. “Carta de los Derechos Fundamentales de la Unión Europea”. DO C 326/02 de 26.10.2012, p. 391-407.

UNIÓN EUROPEA. “Tratado de la Unión Europea”. (TUE) DO C 326/01 de 26.10.2012, p. 13-45.

UNIÓN EUROPEA. "Tratado de Funcionamiento de la Unión Europea”. (TFUE). Versión consolidada, DO C 326/01 de 26.10.2012, p. 47-199. ${ }^{44}$

VALCÁRCEL, Amelia (2000): La memoria colectiva y los retos del feminismo. En, Valcárcel, A. y Romero, R. (eds.). Los desafíos del feminismo ante el siglo XXI. Col. Hypatia, p. 19-54. Sevilla: Instituto Andaluz de la Mujer. Disponible en: http://webs.uvigo.es/pmayobre/pdf/3text_paridad_ya.pdf [actualizado: Julio, 16, 2013]

VALCÁRCEL, Amelia (2004): La política de las mujeres. $3^{\circ}$ ed. Madrid: Cátedra.

WOLLSTONECRAFT, Mary (1994): Vindicación de los derechos de la mujer. Madrid: Cátedra, Universitat de València, Instituto de la Mujer.

YOUNG, Iris Marion (2000): La justicia y la política de la diferencia. Madrid: Ediciones Cátedra, Instituto de la Mujer; Valencia: Universitat de València.

YOUNG, Iris Marion (2011): Responsabilidad por la justicia. Madrid: Ediciones Morata; A Coruña: Fundación Paideia Galiza.

\footnotetext{
${ }^{44}$ Versión consolidada de los tratados y la carta, disponibles en: http://eur-lex.europa.eu/legalcontent/ES/TXT/PDF/?uri=OJ:C:2012:326:FULL\&from=ES [actualizado: Julio, 20, 2014]. 